\title{
Psychometric testing of the World Organization Quality of Life -Bref (WHOQoL-BREF) questionnaire - Farsi version with oncology patients
}

Masoud Bahrami

Abstract: The aim of the study was to provide some evidences for reliability and validity of the WHOQoLBREF questionnaire - Farsi version in Iranian cancer patients. This descriptive correlational study was conducted in 2013 in which 198 cancer patients were conveniently recruited. The Cronbach's alpha values for patients' responses were $0.84,0.75,0.69$, and 0.77 , for physical, psychological, social relationship and environmental domains, respectively. Cronbach alpha values of patients QoL domain scores were above the acceptable level of 0.70 . Most questions $(21 / 24$ or 88 percent) showed the highest correlations with domains to which they generally assigned supporting the for domain structure of the questionnaire. Findings of psychometric testing were generally promising indicating that the WHOQoL-BREF questionnaire was an appropriate tool to use in the clinical area of cancer patients.

Kew words: WHOQoL-BREF questionnaire,

Psychometric testing, oncology patients, Quality of Life,

Masoud Bahrami,

Associate Professor, Department of Adult Health Nursing, Nursing

\& Midwifery Care Research Center, School of Nursing \&

Midwifery, Isfahan University of Medical

Sciences, Isfahan, Iran

\section{Introduction}

The review on the literature shows that quite a number of Quality of Life (QoL) tools exist[1]. This number of QoL tools might indicate that generally there is no need to develop new QoL tools any more. Having a large number of QoL tools can also bring with it the difficulty of making a choice [2]. However, there are several reasons why the World Organization Quality of Life -Bref (WHOQoLBREF) questionnaire Farsi version has initially been chosen for this research study from generic QoL tools. Firstly, it provides a broad assessment of QoL. Ssecondly does not overbore patients and health care professionals who may be asked to complete the questionnaire; and thirdly, is culturally adapted for use in different countries including Iran [3]. However, the most important reason for selecting this questionnaire is having good psychometric properties.

As the WHO group [4] stated, the internal consistency of the WHOQoL-BREF ranges from 0.66 in the social relationship domain to 0.84 in the physical domain and test-retest reliability of the tool 
is 0.75 for all domains. All above correlations fall within intervals $0.61-0.80$ and $0.81-1.00$ which indicate almost substantial and perfect associations, respectively. A population based study in Iran also provided some preliminary evidences of psychometric properties of the Iranian version of the WHOQoL-BREF questionnaire [5]. While it has been concluded that the questionnaire would be useful to measure QoL, the reliability of the social relationship domain and the structure of domains of the questionnaire challenged and recommended to investigated further [5]. Contrary, in another study with the WHOQoL-BREF questionnaire in Iran, findings supported the four domain structure of the questionnaire and its appropriate reliability [6]. However, findings of this study also indicated that some items of the questionnaire loaded on different domains as what originally was proposed for the questionnaire[6]. Due to these controversies, it was the aim of the study to provide some evidences for reliability and validity of this questionnaire in cancer patients using the internal consistency test and exploratory factor analysis.

\section{Material and methods}

This descriptive correlational study was a part of a bigger QoL research which was conducted during a six month period in 2013 in Syeed-Al -Shohada hospital affiliated to Isfahan University of Medical
Sciences, Isfahan, Iran. Other part of the results published somewhere else [7]. In order to measure psychometric properties of the WHOQoL-BREF questionnaire - Farsi version, 198 cancer patients were conveniently recruited. The WHOQoL-BREF questionnaire - Farsi version was used for this research. This test uses 26 items which assess the QoL for four domains or dimensions, including physical (7 items), psychological (6 items), social relationship (3 items), and environmental (8 items) domains, and 2 items measuring overall quality of life and general health [4].

Patients were eligible for inclusion in the study if they had confirmed diagnosis of any kind of cancer; reached the age of 18 years or older; and had the ability to read and write in Farsi (Persian) to be able to respond to the questionnaire appropriately; and agreed to participate in the study. Patients were selected from all inpatient and outpatient oncology centers and differed in their health status, disease severity and treatment modalities.

\section{Results}

The Cronbach's alpha values for patients' responses were $0.84,0.75,0.69$, and 0.77 , for physical, psychological, social relationship and environmental domains, respectively. To explore the structure of the WHOQoL-BREF questionnaire completed by patients, Principal Components Analysis (PCA) was 
conducted by SPSS version 12 software using the method as suggested by Pallant[8]. Kaiser-MeyerOlkin measure of sampling adequacy was 0.87 , exceeding the recommended value of 0.60 . The Bartlett's test of Sphericity reached statistical significance, supporting the factorability of the correlation matrix for patients. Varimax rotation was conducted to see how items of the questionnaire will be loaded on a four domain structure.

\section{Discussion}

Results of the reliability test using internal consistency identified that the Cronbach alpha values of patients QoL domain scores were above the acceptable level of 0.70 . However, results of construct validity using an exploratory factor analysis need to be taken also into the consideration. Similar to other research findings [5] most questions (21/24 or 88 precent) showed the highest correlations with domains to which they generally assigned. However, three items including "conditions of your living place", "healthy physical environment" and "safe in your daily life" from the environmental domain, strongly loaded on the social relationship domain. As stated in another study in Iran [6] such findings might indicate that these items were considered by cancer patients more as social concerns rather than environmental issues for several reasons. For example, patients might personally accept deficiencies in resources and facilities. However, how such incomes and services equally distributed might be a concern for patients.

Altogether, findings of psychometric testing were promising indicating that the WHOQoL-BREF questionnaire was an appropriate tool to use in the clinical area of cancer patients. However, still some small modifications might be necessary to conduct in social and/ or environmental items to improve this tool to a more acceptable level for a QoL assessment in an Iranian context.

\section{Acknowledgment}

The Nursing and Midwifery Care Research Centre of Isfahan University of Medical Sciences supported this study financially (Grant No. 289301). I want to thank all of the patients of Seyed-Al-Shohada Hospital that gave consent to participate in this study.

\section{References}

[1] Salek, M.S., ed. Compendium of quality of life instruments. 1998, John Wiley \& Sons: New York. 5 v.

[2] Bahrami, M., quality of life in cancer and other chronic diseases. 2012: Isfahan University of Medical Sciences.

[3] Bahrami, M., Do nurses provide holistic care to cancer patients? Iran J Nurs Midwifery Res, 2010. 15(4): p. 245-51.

[4] The WHO Group, Development of the World Health Organization WHOQOL-BREF Quality of Life Assessment. . Psychological Medicine, 1998. 28(3): p. 551-558.

[5] Nedjat, S., et al., Psychometric properties of the Iranian interview-administered version of the World Health Organization's Quality of Life Questionnaire (WHOQOLBREF): a population-based study. BMC Health Serv Res, 2008. 8: p. 61.

[6] Yousefy, A.R., et al., Psychometric properties of the WHOQOL-BREF in an Iranian adult sample. Community Ment Health J, 2010. 46(2): p. 139-47.

[7] Bahrami, M., Iranian Nurses Perceptions of Cancer Patients Quality of Life. Iran J Cancer Prev, 2016. 9(3): p. e4076.

[8] Pallant, J.F., SPSS survival manual: a step by step guide to data analysis using SPSS. 2nd ed. 2004, Crows Nest, N.S.W.: Allen \& Unwin. xvi, 318. 
Proc. of The Sixth Intl. Conf. On Advances In Economics, Social Science and Human Behaviour Study - ESSHBS 2017 Copyright $\odot$ Institute of Research Engineers and Doctors, USA .All rights reserved.

ISBN: 978-1-63248-120-7 doi: 10.15224/ 978-1-63248-120-7-75

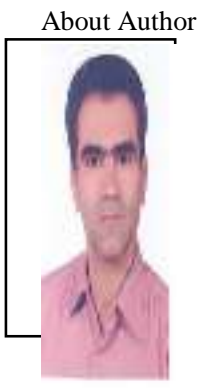

BSN,MSN, PhD in Med- Surg Nursing,

Associate Professor in School of Nursing and Midwifery, Isfahan University of

Medical Sciences, Isfahan, Iran. 